\title{
Combination therapy with lentinan improves outcomes in patients with esophageal carcinoma
}

\author{
JI-LIAN WANG $^{1 *}$, ZHENG BI $^{2 *}$, JIAN-WEN ZOU ${ }^{3}$ and XIAO-MENG GU ${ }^{4}$ \\ ${ }^{1}$ Department of Clinical Laboratory, Dezhou People's Hospital, Dezhou, Shandong 253014; \\ ${ }^{2}$ Department of Clinical Laboratory, Qilu Hospital, Shandong University, Jinan, Shandong 250012; \\ ${ }^{3}$ Department of Clinical Laboratory, Shandong Provincial Hospital, Shandong University, Jinan, Shandong 250012; \\ ${ }^{4}$ Department of Gastroenterology, Qilu Hospital, Shandong University, Jinan, Shandong 250012, P.R. China
}

Received September 14, 2011; Accepted November 10, 2011

DOI: $10.3892 / \mathrm{mmr} .2011 .718$

\begin{abstract}
With a view to improving treatment response and the quality of life of cancer patients, this study investigated the clinical efficacy of combining lentinan, a flavonoid compound with antitumor abilities, with traditional chemotherapy in individuals with esophageal carcinoma (EC), with a particular focus on its effect on immune function. A total of 50 patients undergoing treatment for EC were evenly divided into two groups: control and experimental. Patients in the control group were treated with the chemotherapeutic agent tegafur (1,000 mg/day for 5 days); patients in the experimental group were treated with the same dosage of tegafur combined with $1 \mathrm{mg}$ lentinan diluted in $250 \mathrm{ml}$ normal saline. Patients were monitored for their general condition, symptoms and signs, quality of life and clinical efficacy (remission vs. progression). Additionally, the effects of lentinan on immune function were assessed through analysis of serum levels of pro-inflammatory and anti-inflammatory cytokines using enzyme-linked immunosorbent assay (ELISA) prior to and following the first and second course of treatment. The results of the scores showed that the general condition (Karnofsky performance scale; KPS), the symptoms and signs (ZubrodECOG-WHO score; ZPS) and the quality of life (QOL scale) of the patients following the first and second course of treatment were better in both groups compared to the scores prior to treatment; however, patients in the experimental group exhibited significantly greater improvement than those in the control group $(\mathrm{P}<0.05)$. Clinical efficacy was not significantly different between the two groups after 1 course of treat-
\end{abstract}

Correspondence to: Dr Xiao-Meng Gu, Department of Gastroente-rology, Qilu Hospital, Shandong University, Jinan, Shandong 250012, P.R. China

E-mail: xiaomeng914@126.com

${ }^{*}$ Contributed equally

Key words: lentinan, esophageal carcinoma, clinical efficacy, immunological function ment, but after 2 courses of treatment, clinical efficacy was significantly greater in the experimental group than in the control group $(\mathrm{P}<0.05)$. Additionally, serum levels of IL-2, IL-6 and IL-12 increased, while levels of IL-4, IL-5 and IL-10 decreased, in patients of both groups after 2 courses of treatment $(\mathrm{P}<0.05)$. These changes occurred to a greater extent in the experimental group than in the control group $(\mathrm{P}<0.05)$. In conclusion, the addition of lentinan to the chemotherapy regimen improves the general condition, symptoms and signs, and quality of life of patients with EC. In particular, the patient's immune function may be enhanced by the combined treatment. The generalized application of lentinan is, therefore, recommended in the clinic.

\section{Introduction}

Esophageal carcinoma (EC), a malignant tumor affecting the squamous epithelium of the esophagus, occurs commonly in China. Indeed, China has one of the highest incidences of and mortality from this type of cancer: the average annual mortality rate is $14.59 / 100,000$, making it the fourth deadliest malignancy (1). Currently, EC is treated with combined surgery, radiotherapy and chemotherapy. While these treatments present efficacy in treating EC, they also result in toxicity and other side effects (2). Therefore, intensive research into alternative therapies is required to improve the quality of life of patients and to prolong survival time in EC.

Traditional Chinese medicine has unique advantages in the comprehensive treatment of cancer. Several traditional Chinese medicines have antitumor, antimutagenic and immunoregulatory functions (3). A notable example is the compound lentinan, a flavonoid extracted from shiitake mushrooms, which is used particularly in combination with conventional chemotherapy as an adjuvant. A biological-response modifier, lentinan enhances immunity, improves efficacy, reduces toxicity and improves the quality of life during chemotherapy (4). Although lentinan has been applied to the treatment of colon, gastric and liver cancers, no evidence exists for its efficacy as an adjuvant in the treatment of EC. Since EC is a common and fatal disease, we sought to explore the clinical efficacy of lentinan combined with chemotherapy in patients with EC. Furthermore, we investigated the effect of lentinan 
Table I. Scores for the general condition (KPS), the symptoms and signs (ZPS), and the quality of life (QOL) in patients with EC following treatment with tegafur (control group) or tegafur plus lentinan (experimental group).

\begin{tabular}{|c|c|c|c|c|c|c|c|}
\hline Scores & Group & $\mathrm{n}$ & Before treatment & After 1 course & After 2 courses & $\mathrm{F}$ & P-value \\
\hline \multirow[t]{2}{*}{ KPS score ${ }^{a}$} & Control & 25 & $45.20 \pm 5.86$ & $51.60 \pm 7.46$ & $58.40 \pm 9.87$ & 45.284 & 0.001 \\
\hline & Experimental & 25 & $47.20 \pm 5.42$ & $56.40 \pm 4.90$ & $64.80 \pm 9.63$ & 108.298 & 0.001 \\
\hline \multirow[t]{2}{*}{ ZPS score ${ }^{b}$} & Control & 25 & $2.32 \pm 0.48$ & $2.20 \pm 0.41$ & $1.80 \pm 0.41$ & 17.935 & 0.001 \\
\hline & Experimental & 25 & $2.20 \pm 0.41$ & $1.84 \pm 0.37$ & $1.44 \pm 0.51$ & 23.312 & 0.001 \\
\hline \multirow[t]{2}{*}{ QOL score } & Control & 25 & $34.56 \pm 3.06$ & $39.92 \pm 1.78$ & $42.44 \pm 8.79$ & 14.177 & 0.001 \\
\hline & Experimental & 25 & $34.92 \pm 2.27$ & $41.60 \pm 2.53$ & $48.60 \pm 2.16$ & 324.659 & 0.001 \\
\hline
\end{tabular}

Inter-group comparison: ${ }^{\mathrm{a}} \mathrm{F}=5.821, \mathrm{P}=0.020 ;{ }^{\mathrm{b}} \mathrm{F}=9.692, \mathrm{P}=0.003 ;{ }^{\mathrm{c}} \mathrm{F}=13.446, \mathrm{P}=0.001$.

Table II. Clinical efficacy of lentinan in patients with esophageal carcinoma [n (\%)].

\begin{tabular}{lcccccc}
\hline Response time & Group & $\mathrm{n}$ & Partial remission & Improvement & Disease stability & Disease progression \\
\hline${\text { After } 1 \text { course }^{\mathrm{a}}}$ & Control & 25 & $1(4.0)$ & $3(12.0)$ & $17(68.0)$ & $4(16.0)$ \\
& Experimental & 25 & $2(8.0)$ & $5(20.0)$ & $18(72.0)$ & 0 \\
${\text { After } 2 \text { courses }^{\mathrm{b}}}$ & Control & 25 & $2(8.0)$ & $4(16.0)$ & $17(58.0)$ & $2(8.0)$ \\
& Experimental & 25 & $4(16.0)$ & $9(36.0)$ & $12(48.0)$ & 0 \\
\hline
\end{tabular}

Inter-group comparison: ${ }^{\mathrm{a}} \mathrm{U}=1.778, \mathrm{P}=0.075 ;{ }^{\mathrm{b}} \mathrm{U}=2.156, \mathrm{P}=0.031$.

with chemotherapy on host immune function by assaying serum cytokines IL-2, IL-6, IL-12, IL-4, IL-5 and IL-10.

\section{Patients and methods}

Patients. Fifty EC patients from the Department of Gastroenterology, Qilu Hospital, Shandong University, diagnosed according to the World Health Organization (WHO) reference criteria, were enrolled in this study. The patients were divided evenly into a control group $[\mathrm{n}=25,16$ males, 9 females; 24-72 years of age (mean 59.8 \pm 10.1 )] and an experimental group [n=25, 14 males, 11 females; 26-75 years of age (mean 60.8 \pm 9.6 )], according to their order of hospitalization. No significant differences were detected in gender, age, general condition, symptoms and signs, or quality of life $(\mathrm{P}>0.05)$ between these groups.

Therapeutic methods. Patients in the control group were treated continuously for 5 days with $1,000 \mathrm{mg} /$ day tegafur (trade name Fangke, produced by Shandong Qilu Pharmaceutical Co., Ltd., Jinan, China) by intravenous (i.v.) drip. Patients in the experimental group received the same dosage of tegafur, plus $1 \mathrm{mg}$ lentinan (National Medicine permit no. Z10920011; Fuzhou Meifeng Pharmaceutical Factory of Jinling Pharmaceutical Co., Ltd., Nanjing) diluted in $250 \mathrm{ml}$ normal saline, administered every other day. Treatment occurred in 2 courses over 21 days.

Outcome measures. The Karnofsky performance scale score (KPS score, percentages), The Zubrod-ECOG-WHO score (ZPS score, 5-point method) and the QOL scale (12 grades,
60 points) were used to assess the general condition, the symptoms and signs, and the quality of life of the patients, respectively, prior to and following each of the 2 treatment courses. Clinical efficacy was determined before and after the two courses of treatment using WHO objective efficacy criteria for solid tumor treatment (5); the following five grades were included: complete remission, partial remission, improvement, disease stability and disease progression. The double-antibody sandwich ELISA (DAS-ELISA) was used to detect serum IL-2, IL-6, IL-12, IL-4, IL-5 and IL-10 levels, according to the manufacturer's instructions (R\&D Systems, Minneapolis, MN, USA).

Statistical methods. SPSS13.0 statistical software was used for statistical analysis; all results are expressed as the means \pm standard deviation. Analysis of variance with repeated measures was used to compare differences in the general condition, the signs and symptoms, and the quality of life scores, as well as the serum cytokine levels, between the two groups. Non-parametric Mann-Whitney U test was used to compare efficacy between groups. Analysis was performed with a two-sided test, at $\alpha$ level 0.05 , with $\mathrm{P}<0.05$ considered to be statistically significant.

\section{Results}

Lentinan therapy improves outcomes in patients with EC. The clinical condition of patients with EC was assessed prior to chemotherapy, as well as following each course of treatment. Scores reflecting the general condition (KPS), the symptoms and signs (ZPS) and the quality of life (QOL) of the patients 

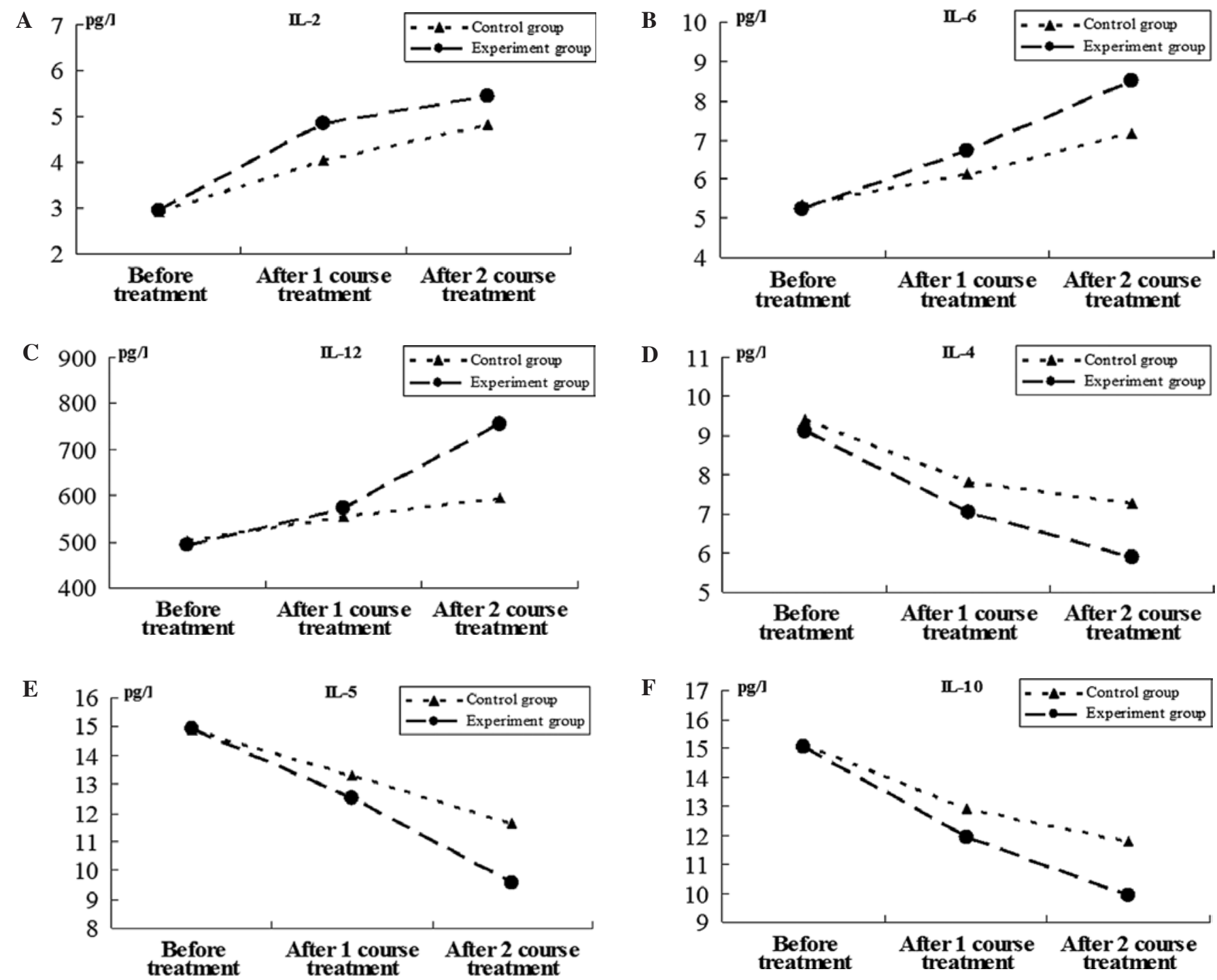

Figure 1. Serum cytokine levels in patients with esophageal carcinoma. (A) IL-2 levels: $\mathrm{F}_{\text {Experimental group }}=38.045, \mathrm{P}=0.001 ; \mathrm{F}_{\text {Control group }}=62.230, \mathrm{P}=0.001$; $\mathrm{F}_{\text {Experimental vs. control }}=16.616, \mathrm{P}=0.001$. (B) IL-6 levels: $\mathrm{F}_{\text {Experimental group }}=182.147, \mathrm{P}=0.001 ; \mathrm{F}_{\text {Control group }}=85.463, \mathrm{P}=0.001 ; \mathrm{F}_{\text {Experimental vs. control }}=10.206, \mathrm{P}=0.002$. (C) IL-12 levels: $\mathrm{F}_{\text {Experimental group }}=37.007, \mathrm{P}=0.001 ; \mathrm{F}_{\text {Control group }}=6.323, \mathrm{P}=0.004 ; \mathrm{F}_{\text {Experimental vs control }}=11.306, \mathrm{P}=0.002 .(\mathrm{D}) \mathrm{IL}-4$ levels: $\mathrm{F}_{\text {Experimental group }}=24.224$, $\mathrm{P}=0.001 ; \mathrm{F}_{\text {Control group }}=10.282, \mathrm{P}=0.001 ; \mathrm{F}_{\text {Experimental vs. control }}=9.989, \mathrm{P}=0.003$. (E) IL-5 levels: $\mathrm{F}_{\text {Experimental group }}=28.963, \mathrm{P}=0.001 ; \mathrm{F}_{\text {Control group }}=10.079, \mathrm{P}=0.001 ;$ $\mathrm{F}_{\text {Experimental vs. control }}=5.110, \mathrm{P}=0.003$. (F) IL-10 levels: $\mathrm{F}_{\text {Experimental group }}=538.848, \mathrm{P}=0.001 ; \mathrm{F}_{\text {Control group }}=69.048, \mathrm{P}=0.001 ; \mathrm{F}_{\text {Experimental vs. control }}=10.206, \mathrm{P}=0.002$.

following each of the 2 treatment courses, were significantly better than scores prior to treatment for patients in both the control (tegafur only) and experimental (tegafur plus lentinan) group $(\mathrm{P}<0.05$; Table I). However, improvement in each of these measures was greater in the experimental group than in the control group $(\mathrm{P}<0.01)$.

Clinical efficacy of each treatment paradigm was determined based on the WHO criteria for solid tumor treatment and included the following five grades: complete remission, partial remission, improvement, stability and disease progression (Table II). Following 1 course of tegafur alone (control), we observed 1 case with partial remission, 3 cases with improvement, 17 cases with disease stability and 4 cases with disease progression. Following 1 course of tegafur combined with lentinan (experimental), we observed 2 cases with partial remission, 5 cases with improvement and 18 cases with disease stability. However, these differences were not statistically significant. Following 1 treatment course, there were 2 cases with partial remission, 4 cases with improvement, 17 cases with disease stability and 2 cases with disease progression in the control group, and 4 cases with partial remission, 9 cases with improvement and 12 cases with disease stability in the experimental group. In contrast to the outcomes following 1 course of treatment, clinical efficacy was significantly better following 2 courses of tegafur plus lentinan than tegafur alone $(\mathrm{U}=2.156, \mathrm{P}=0.0031)$.

Serum cytokine levels are altered following treatment for $E C$. To determine the mechanism behind the enhanced improvement following combined therapy, we investigated the immune responses of the patients by assessing the levels of serum cytokines. Serum cytokines in patients with EC were detected using DAS-ELISA prior to and following each treatment course. Serum IL-2, IL-6 and IL-12 levels increased (Fig. 1A-C), while serum IL-4, IL-5 and IL-10 levels decreased (Fig. 1D-F) in patients of both groups following each course 
of treatment compared to levels prior to treatment $(\mathrm{P}<0.05)$. Of note, these changes in serum cytokine levels were greater with the lentinan treatment (experimental group) than without (control group; $\mathrm{P}<0.05$ ).

\section{Discussion}

Decreased immune function is a major factor leading to the occurrence of malignant tumors. Antitumor immunity is mainly cell-mediated immunity, in which $\mathrm{T}$ lymphocytes play a central regulatory role (6). Lentinan, a glucan isolated and purified from mushrooms, is an immune enhancer that affects the function of $\mathrm{T}$ cells and macrophages, promotes proliferation of $\mathrm{T}$ and $\mathrm{B}$ lymphocytes, and increases activity of natural killer (NK) cells $(4,7)$. Thus, the application of lentinan in cancer therapy enhances host immunity and tumor-fighting ability. Indeed, in combining lentinan with traditional chemotherapy in patients with $\mathrm{EC}$, we observed greater improvement in the general condition, the signs and symptoms, and the quality of life over traditional chemotherapy alone. Additionally, the clinical efficacy (assessed as tumor remission, stability or progression) in patients receiving lentinan in addition to traditional chemotherapy was significantly better than traditional chemotherapy alone after just 2 courses of treatment. Thus, the addition of lentinan to the chemotherapy regimen improves the quality of life and the outcomes of patients with EC.

To determine the mechanism by which lentinan treatment results in better outcomes in EC therapy, we investigated the immune function by assessing serum cytokines. Cytokines have pro- or anti-inflammatory activities (or both). Pro-inflammatory cytokines such as IL-2, IL-6 and IL-12 help activate the immune response and therefore minimize tumor development or progression. IL-2 participates in the inflammatory response and has antitumor effects (8). IL-6 has been shown to affect occurrence and development of EC, fighting tumors through mobilization of antitumor immune mechanisms or by direct effects on cancer cells (9). IL-12 activates NK cells, enhances PHA-induced lymphoblast proliferation, promotes synthesis of pro-inflammatory cytokines IFN- $\beta$ and TNF- $\alpha$ by Th1 cells, and inhibits synthesis of anti-inflammatory cytokines IL-4, IL- 5 and IL- 10 by Th 2 cells (10). By contrast, anti-inflammatory cytokines such as IL-4, IL-5 and IL-10 are immunosuppressive. IL-4 and IL-5 are produced by Th2 cells and are able to inhibit proliferation of Th1 cells and other immune responses (11). IL-10 is a cytokine synthesis inhibitor, a strong immunosuppressive factor with multi-directional biological activities and an ability to change the body's immune response or MHC class II antigen expression and mediate mutual regulation between Th1 and Th2 cells (12). Indeed, in the presence of mononuclear cells, IL-10 directly inhibits $\mathrm{T}$ cell proliferation and cytokine production (13). Furthermore, IL-10 plays an important role in the occurrence of dendritic cell dysfunction and immune escape in patients with EC (14).
In the present study, we showed that the serum levels of pro-inflammatory cytokines IL-2, IL-6 and IL-12 gradually increased following each course of treatment, but particularly for those treated with lentinan as well as tegafur, indicating an up-regulation of the immune response. Concurrent decreases in the serum levels of anti-inflammatory cytokines IL-4, IL-5 and IL-10 support this hypothesis. Thus, when combined with traditional chemotherapy, lentinan improves immune function in patients with EC. This up-regulation of the immune response may be responsible for the larger improvements in the clinical condition/quality of life and efficacy in these patients compared to those treated with traditional chemotherapy alone.

In conclusion, lentinan improves the general condition, the signs and symptoms, and the quality of life of patients with EC and improves their clinical outcomes. Lentinan acts by up-regulating pro-inflammatory cytokines and down-regulating anti-inflammatory cytokines. These positive results, combined with the safety and the lack of apparent side effects of this treatment, render lentinan a promising adjuvant chemotherapy.

\section{References}

1. Jemal A, Siegel R, Xu J and Ward E: Cancer statistics, 2010. CA Cancer J Clin 60: 277-300, 2010.

2. Mariette C and Triboulet JP: What's new in esophageal carcinoma treatment. Presse Med 36: 496-500, 2007.

3. Yang H, Liu J and Dou QP: Targeting tumor proteasome with traditional Chinese medicine. Curr Drug Discov Technol 7: 46-53, 2010.

4. Yoshino S, Watanabe S, Imano M, et al: Improvement of QOL and prognosis by treatment of superfine dispersed lentinan in patients with advanced gastric cancer. Hepatogastroenterology 57: 172-177, 2010.

5. Watanabe H, Okada M, Kaji Y, et al: New response evaluation criteria in solid tumours-revised RECIST guideline (version 1.1). Gan To Kagaku Ryoho 36: 2495-2501, 2009.

6. Seliger B: Strategies of tumor immune evasion. Bio Drugs 19: 347-354, 2005.

7. Wang X, Zhang Y, Zhang L and Ding Y: Multiple conformation transitions of triple helical lentinan in $\mathrm{DMSO} /$ water by microcalorimetry. J Phys Chem B 113: 9915-9923, 2009.

8. Foa R, Guarini A and Gansbacher B: IL2 treatment for cancer: from biology to gene therapy. Br J Cancer 66: 992-998, 1992.

9. Bennermo M, Held C, Stemme S, et al: Genetic predisposition of the interleukin- 6 response to inflammation: implications for a variety of major diseases? Clin Chem 50: 2136-2140, 2004.

10. Li XH, Ma J, Wu XX, et al: Effect of various combinations of IL2, IL12 and IL15 on function of human peripheral blood derived NK cells. Zhongguo Shi Yan Xue Ye Xue Za Zhi 17: 918-923, 2009.

11. Codlin S, Soh C, Lee T and Lavender P: Characterization of a palindromic enhancer element in the promoters of IL4, IL5, and IL13 cytokine genes. J Allergy Clin Immunol 111: 826-832, 2003.

12. Fujio K, Okamura T and Yamamoto K: The family of IL-10secreting CD4 ${ }^{+} \mathrm{T}$ cells. Adv Immunol 105: 99-130, 2010.

13. Saraiva M and O'Garra A: The regulation of IL-10 production by immune cells. Nat Rev Immunol 10: 170-181, 2010.

14. Yang WF, Yu JM, Zuo WS and Wang SZ: Expression of CD80, CD86, TGF-betal and IL-10 mRNA in the esophageal carcinoma. Zhonghua Zhong Liu Za Zhi 28: 762-765, 2006. 